\title{
Occurrence and fate of perfluoroalkyl substances in marine sediments from the Chinese Bohai Sea, Yellow Sea, and East China Sea
}

\author{
Yan Gao a , Jianjie Fu ${ }^{a}$, Lixi Zeng ${ }^{b}$, An Li ${ }^{c}$, Huijuan Li ${ }^{a}$, Nali Zhu ${ }^{a}$, Runzeng Liu ${ }^{a}$, \\ Aifeng Liu ${ }^{\text {a }}$, Yawei Wang ${ }^{\text {a, }}$, Guibin Jiang ${ }^{\text {a }}$ \\ a State Key Laboratory of Environmental Chemistry and Ecotoxicology, Research Center for Eco-Environmental Sciences, Chinese Academy of Sciences, \\ P.O. Box 2871, Beijing 100085, China \\ ${ }^{\mathrm{b}}$ School of Chemistry and Chemical Engineering, University of Chinese Academy of Sciences, Beijing 100049, China \\ ${ }^{c}$ School of Public Health, University of Illinois at Chicago, Chicago, USA
}

\section{A R T I C L E I N F O}

\section{Article history:}

Received 26 April 2014

Received in revised form

3 July 2014

Accepted 10 July 2014

Available online 4 August 2014

\section{Keywords:}

Perfluoroalkyl substances

Marine sediment

Spatial distribution

Temporal distribution

\begin{abstract}
A B S T R A C T
In this study, 166 surface sediments and 3 sediment cores from the Bohai Sea (BS), Yellow Sea (YS) and East China Sea (ECS) in China were collected to investigate the spatial and temporal distributions and the transport of PFASs. PFASs concentrations in the surface sediments ranged from below detection limit $(<\mathrm{LOD})$ to $2.78 \mathrm{ng} \mathrm{g}^{-1}$ with an average value of $0.55 \mathrm{ng} \mathrm{g}^{-1}$ on a dry weight basis (dw). A general decreasing trend of PFASs from the coast areas to the open sea was found. Multivariate regression analysis indicated $\mathrm{pH}$ and longitude were the major factors influencing surficial distributions of PFASs in the sampling areas $\left(R^{2}=0.29, p<0.01\right)$. Total PFASs concentrations in the sediment cores ranged from $<$ LOD to $1.65 \mathrm{ng} \mathrm{g}^{-1} \mathrm{dw}$, with an increasing trend from the lower to the upper layers, corresponding well to the increasing production and usage in China in recent years.
\end{abstract}

(C) 2014 Elsevier Ltd. All rights reserved.

\section{Introduction}

Per- and polyfluoroalkyl Substances (PFASs), such as perfluorocarboxylates (PFCAs) and perfluorosulfonates (PFSAs), have been widely used in the past six decades as additives in surface coatings of carpets, leather, papers, and textiles, as well as in firefighting foams, pesticides, paints, etc. Their excellent thermal and chemical stability renders PFASs highly persistent in the environment, evidenced by their ubiquitous global detection (Cai et al., 2012b) and accumulations in human and wildlife (Kelly et al., 2009; Li et al., 2013). In 2009, perfluorooctanesulfonate (PFOS) and its salts were listed in the Stockholm Convention as Persistent Organic Pollutants (POPs) (Loi et al., 2011).

Production and usage of PFASs in China has increased rapidly in recent years. A large proportion of the total production output in China was from coastal provinces/cities, including Zhejiang, Fujian, Guangdong, Jiangsu, Liaoning and Shanghai (Chen et al., 2009), adjacent to the Bohai Sea (BS), Yellow Sea (YS), East China Sea (ECS), and South China Sea (SCS). Recently, PFASs have been detected in the coastal marine environment of China. Chen et al. investigated

\footnotetext{
* Corresponding author.

E-mail address: ywwang@rcees.ac.cn (Y. Wang).
}

PFASs in the water and sediment from coastal northern BS and found sediment was an important sink for perfluoroundecanoic acid (Chen et al., 2011). Pan et al. found low PFASs concentrations in mollusks from the coastal waters in the BS (Pan et al., 2010). The investigation on the PFASs in coastal waters of Hong Kong, South China indicated that PFASs in the sampling areas were strongly influenced by discharge from the Pearl River (So et al., 2004). Cai et al. considered the Yangtze River as an important source of PFASs found in the coastal waters of southern China (Cai et al., 2012a).

In spite of the ubiquitous existence of PFASs in the global environment, the mechanisms of long range transport of the PFASs and their precursors across continents and on global scales remain unclear. Relatively volatile perfluoroalkyl precursors such as fluorotelomer alcohols (FTOHs), perfluoroalkyl sulfonamides (FASAs) and perfluoroalkylsulfonamidoethanols (FASEs) were transported via the atmosphere and subject to photolytic degradation in the air (Ellis et al., 2004, 2003), whereas the ionisable PFASs such as PFCAs and PFSAs are predominantly transported via the ocean current (Prevedouros et al., 2006).

This work is a systematic survey of PFASs in the sediments of the BS, YS and ECS of China. The primary objective of this work was to depict the spatial and temporal distribution patterns of PFASs in the three sea regions of China. The large number of samples allowed us to examine the factors influencing the accumulation of PFASs in 
marine sediments. The large geographical coverage enabled us to gain insights into the anthropogenic impacts, the transport pathways, and the environmental fate of PFASs in the sampling areas.

\section{Materials and methods}

\subsection{Sample collection}

The entire study area encloses the BS, YS and ECS, extending $2000 \mathrm{~km}$ from south to north and totaling about $1,230,000 \mathrm{~km}^{2}$ water surface (Fig. 1). The major mud areas and the dominant ocean currents are shown in Fig. S1. The BS is a semienclosed shallow (mean depth $26 \mathrm{~m}$ ) marginal sea receiving waters from more than 40 rivers in northern China. The YS is located between Chinese mainland to the west and Korean Peninsula to the east, with an average depth of $44 \mathrm{~m}$. ECS is mostly on a continental shelf with water depth $<200 \mathrm{~m}$, and its water flow is strongly influenced by the freshwater input from the Yangtze River and the saline Kuroshio Current (Yanao and Matsuno, 2013). The YS and ECS constitute the western margin of the North Pacific Ocean.

Sediment sampling was carried out onboard of R/V Dong Fang Hong 2 in 2011 and 2012. The sampling locations are shown in Fig. 1, and additional information is summarized in Table S1. In all, 166 surface sediments were collected using a stainless steel box sampler and transferred to polyethylene bags. Three sediment cores were collected. Core- 1 and Core-2 were collected in the ECS in 2011, and Core-3 was in the YS in 2012, all from the mud areas of the shelf. The sediment cores were sectioned onboard into $1 \mathrm{~cm}$ intervals using a stainless steel cutter. A total of 111 core slices were obtained. All samples were kept in a shipboard refrigerator immediately after sampling, and subsequently freeze-dried and ground after being transported to the laboratory. All the samples were stored frozen at $-20{ }^{\circ} \mathrm{C}$ until analysis.

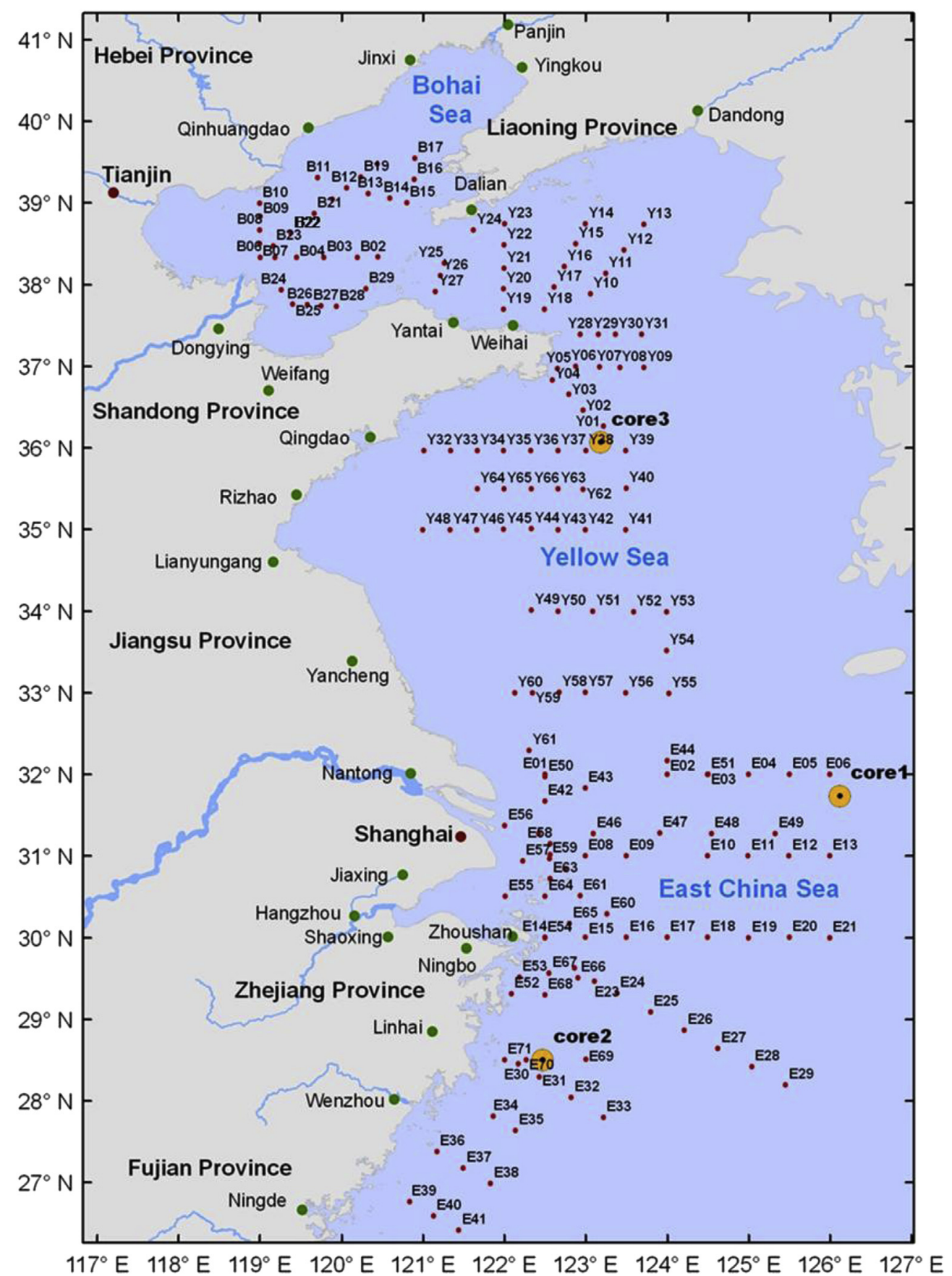

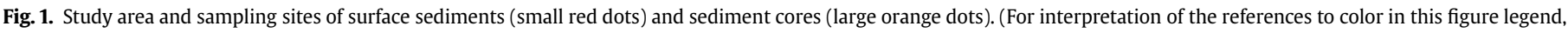
the reader is referred to the web version of this article.) 


\subsection{Chemicals and reagents}

A mixture (PFAC-MXB) of 6 PFCAs $\left(C_{7}-C_{12}\right)$ and 3 PFSAs $\left(C_{4}, C_{6}, C_{8}\right)$ was purchased from Wellington Laboratories (Canada). Carbon-13 labeled ${ }^{13} \mathrm{C}_{4} \mathrm{PFOS}$ and ${ }^{13} \mathrm{C}_{4} \mathrm{PFOA}$ were also purchased from Wellington. Methanol (HPLC grade) was purchased from J.T.Baker (USA). Ammonium acetate was purchased from Alfa Aesar (Ward Hill, MA, USA). Water was prepared by a Milli-Q Advantage A10 system (Millipore Corp., USA). Oasis-HLB cartridge (6 cc, $150 \mathrm{mg}$ ), made in Ireland, was purchased from Waters Corp. (Milford, MA, USA).

\subsection{Sample preparation and instrumental analysis}

$5 \mathrm{~g}$ of sediment samples was weighed into a $50-\mathrm{mL}$ polypropylene tube and spiked with internal standards $\left({ }^{13} \mathrm{C}_{4}\right.$ PFOS and ${ }^{13} \mathrm{C}_{4} \mathrm{PFOA}, 5 \mathrm{ng}$ each). $20 \mathrm{~mL}$ of methanol was added as extracting agent. The mixture was sonicated for $20 \mathrm{~min}$ and centrifuged at $3500 \mathrm{rpm}$ for $10 \mathrm{~min}$. The supernatant was transferred into a new tube. The remnant solution was extracted twice by shaking with $10 \mathrm{~mL}$ of methanol. The extracts were concentrated to $1 \mathrm{~mL}$ under a steam of $\mathrm{N}_{2}$.

Solid phase extraction (SPE) was used for sample cleanup. The Oasis-HLB SPE cartridge was preconditioned with $7 \mathrm{~mL}$ of methanol and $7 \mathrm{~mL}$ of Mili-Q water. The concentrated extract was diluted with $40 \mathrm{~mL}$ deionized water and loaded on the preconditioned cartridge by gravity, after which $5 \mathrm{~mL}$ of methanol/water (1:4) was loaded. Target analytes were eluted by $10 \mathrm{~mL}$ methanol. The elution was concentrated to $1 \mathrm{~mL}$ by gentle stream of $\mathrm{N}_{2}$ and transferred to an injection vial.

Analysis of PFASs was performed using a HPLC-ESI-/MS/MS system consisted of a Waters 2695 Alliance high performance liquid chromatography and a Waters Quattro Premier XE triple-quadrupole mass spectrometer (Waters Corp., Milford, MA). For each run, $10 \mathrm{uL}$ of the sample was separated on a Dionex Acclaim 120 C18 column ( $5 \mu \mathrm{m}, 4.6 \mathrm{~mm}$ i.d. $\times 150 \mathrm{~mm}$ length; Dionex, Sunnyvale, CA, USA). Methanol (A) and $10 \mathrm{mM}$ ammoniumacetate aqueous solution (B) were used as mobile phases. The flow rate was $1.0 \mathrm{~mL} \mathrm{~min}^{-1}$. The dualistic gradient started at $28 \% \mathrm{~B}$, changed to $5 \%$ $\mathrm{B}$ in 4 min linearly, returned to $28 \% \mathrm{~B}$ in 3 min linearly, and then balanced for $3 \mathrm{~min}$. The precursor and product ions were listed in Table S1.

\subsection{Quality assurance/quality control}

The method limit of quantification (MLQ) was determined as ten times of the signal-to-noise ratio. The MLQs were $0.04 \mathrm{ng} \mathrm{g}^{-1}$ dry weight (dw) for PFBS, $0.01 \mathrm{ng} \mathrm{g}^{-1} \mathrm{dw}$ for PFHxS, PFOS, PFHpA, PFOA, and PFNA, and $0.005 \mathrm{ng} \mathrm{g}^{-1} \mathrm{dw}$ for PFDA, PFUnDA and PFDoDA. Detailed information of the matrix spike recoveries and the Standard Reference Materials (SRMs) results was shown in Table S2 and Table S3. One procedural blank was performed for every batch of 7 samples. All of the 9 target compounds in the blanks were below their respective LODs. More detailed information about the quality assurance and quality control is listed in the Supplementary materials.

\subsection{Total organic carbon (TOC) and pH measurements}

TOC was analyzed by an O.I. analytical solids TOC analyzer (O.I. Analytical, USA). Briefly, about $90 \mathrm{mg}$ of samples were placed into the pre-combusted quartz sample cup. Before the analysis, phosphoric acid $\left(1.0 \mathrm{~mol} \mathrm{~L}^{-1}\right)$ was added to remove the inorganic carbon.

To determine the $\mathrm{pH}$ of sediment, $4 \mathrm{~g}$ of dry sediment was weighed into a $50 \mathrm{~mL}$ polypropylene tube. Then, $10 \mathrm{~mL}$ of $1.0 \mathrm{M} \mathrm{KCl}$ solution was added to the tube, which was then well shaken. After standing for $30 \mathrm{~min}$, the supernatant was taken and its pH was measured by a Thermo Orion 3 Star pH Benchtop meter (Li et al., 2005).

\subsection{Statistical analysis}

Statistical analysis was performed with the software SPSS 17.0. Spearman's rank correlation test was used for correlation analysis and a value of $p<0.05$ was considered to be significant. The multivariate linear regression was performed using the stepwise method.

\section{Results and discussion}

Nine PFASs were detected, including perfluorobutanesulfonate (PFBS), perfluorohexanesulfonate (PFHxS), perfluorooctanesulfonate (PFOS), perfluoroheptanoate (PFHpA), perfluorooctanoate (PFOA), perfluorononanoate (PFNA), perluorodecanoate (PFDA), perfluoroundecanoate (PFUnDA) and perfluorododecanoate (PFDoDA). Their detection rates and concentrations were summarized in Table 1 for surface sediments, with complete data compiled in Table S4. TableS5 presents all concentrations for core samples based on dry weight.

\subsection{Concentration and spatial distribution of PFASs in surface sediments}

In 165 of the 166 individual surface sediment samples, at least one PFAS was detected. The total concentrations of PFASs ( ${ }_{9}$ PFASs) averaged $0.55 \mathrm{ng} \mathrm{g}^{-1} \mathrm{dw}$ with a median of $0.43 \mathrm{ng} \mathrm{g}^{-1}$ $\mathrm{dw}$ and a maximum of $2.98 \mathrm{ng} \mathrm{g}^{-1} \mathrm{dw}$. The spatial distribution of ${ }_{9}$ PFASs in the surface sediments was shown in Fig. 2. The $\sum{ }_{9}$ PFASs in the sediments in the different sampling areas were in the ranking of BS > ECS > YS based on both the mean and median (Table 1). Generally, $\sum_{9}$ PFASs were higher near the coast than in remote areas.

In the BS, the highest $\sum_{9}$ PFASs were $2.98 \mathrm{ng} \mathrm{g}^{-1} \mathrm{dw}$ at site B28, which is close to the shore. No obvious spatial trends of PFASs were found along the sampling transects. As BS is semi-enclosed, the exchanges of the seawater are restricted. This reduces the dissipation of the pollutants. Relatively high concentrations of $\sum{ }_{9}$ PFASs were found near the Yellow River estuary and Liaohe River estuary, reflecting the influence of sources on land. In the YS, the concentrations of $\sum_{9}$ PFASs ranged from $<$ LOD at site $Y 34$ to $2.76 \mathrm{ng} \mathrm{g}^{-1} \mathrm{dw}$ at Y66, which is located in the mud areas. Similar to the BS, the spatial gradient is not obvious in the YS. In the ECS, the highest $\sum_{9}$ PFASs of $1.77 \mathrm{ng} \mathrm{g}^{-1} \mathrm{dw}$ was found at site E52. A decreasing gradient was found from the nearshore locations to the open waters (Fig. 2a). The ECS has a dynamic coastal region (Liu et al., 2007), which is influenced by the input from the Yangtze River.

The Yellow Sea warm current (YSWC) and Yellow Sea coastal current (YSCC) facilitate the movement of fine sediment particles (Fig. S1), resulting in the formation of the mud areas in the central YS and BS. Similarly, the Zhejiang-Fujian coastal current (ZFCC), the Tsushima warm current (TaWC) and the Kuroshio warm current (KWC) contribute to the exchange of the seawater and result in the mud areas along the coast of the ECS (Fig. S1). The mud areas with finer sediment particles have accumulated higher concentrations of PFASs than the non-depositional zones (Fig. 2). Post depositional lateral movement of the sediment, or "focusing", carries the fine sediment to the mud areas (Cheng et al., 2004; Liu et al., 2006). For

Table 1

Concentrations ( $\mathrm{ng} \mathrm{g}^{-1} \mathrm{dw}$ ) and detection frequencies ( $d f$ ) of PFASs in surface sediments.

\begin{tabular}{|c|c|c|c|c|c|c|c|c|c|c|c|c|}
\hline & \multicolumn{4}{|c|}{ Chinese Bohai Sea (BS), $N=29$} & \multicolumn{4}{|c|}{ Yellow Sea (YS), $N=66$} & \multicolumn{4}{|c|}{ East China Sea (ECS), $N=71$} \\
\hline & Range & Median & Mean & $d f(\%)$ & Range & Median & Mean & $d f(\%)$ & Range & Median & Mean & $d f(\%)$ \\
\hline PFBS & $<$ LOD -0.11 & $<\mathrm{LOD}$ & $<\mathrm{MQL}$ & 38 & $<$ LOD -0.10 & $<$ LOD & 0.01 & 18 & $<$ LOD -0.19 & $<$ LOD & 0.01 & 15 \\
\hline PFHxS & $<\mathrm{LOD}-0.02$ & $<$ LOD & $<$ LOD & 3 & $<\mathrm{LOD}-0.09$ & $<$ LOD & $<\mathrm{MQL}$ & 12 & $<\mathrm{LOD}-0.33$ & $<$ LOD & 0.01 & 15 \\
\hline PFOS & $<$ LOD -0.15 & 0.06 & 0.06 & 79 & $<$ LOD -0.40 & 0.07 & 0.09 & 92 & $<$ LOD -0.89 & 0.04 & 0.10 & 77 \\
\hline PFHpA & $<$ LOD -0.04 & 0.02 & 0.02 & 52 & $<$ LOD -0.07 & $<$ LOD & $<\mathrm{MQL}$ & 17 & $<$ LOD -0.06 & $<$ LOD & $<\mathrm{MQL}$ & 13 \\
\hline PFOA & $0.06-2.70$ & 0.28 & 0.54 & 100 & $<\mathrm{LOD}-1.52$ & 0.13 & 0.19 & 95 & $<\mathrm{LOD}-0.87$ & 0.12 & 0.18 & 99 \\
\hline PFNA & $<$ LOD -0.31 & 0.05 & 0.05 & 79 & $<$ LOD -0.39 & 0.06 & 0.08 & 83 & $<$ LOD -0.31 & 0.04 & 0.06 & 70 \\
\hline PFDA & $<\mathrm{LOD}-0.04$ & 0.12 & 0.01 & 62 & $<$ LOD -0.12 & 0.14 & 0.02 & 64 & $<\mathrm{LOD}-0.12$ & 0.03 & 0.03 & 72 \\
\hline PFUnDA & $<$ LOD -0.12 & 0.06 & 0.05 & 76 & $<\mathrm{LOD}-0.23$ & 0.07 & 0.08 & 92 & $<\mathrm{LOD}-0.17$ & 0.06 & 0.06 & 80 \\
\hline PFDoDA & $<$ LOD -0.05 & $<$ LOD & $<\mathrm{MQL}$ & 14 & $<$ LOD -0.1 & $<$ LOD & 0.01 & 35 & $<$ LOD -0.57 & 0.02 & 0.06 & 59 \\
\hline$\sum_{9}$ PFASs & $0.06-2.98$ & 0.58 & 0.77 & 100 & $<$ LOD -2.76 & 0.36 & 0.49 & 98 & $0.03-1.77$ & 0.41 & 0.52 & 100 \\
\hline
\end{tabular}



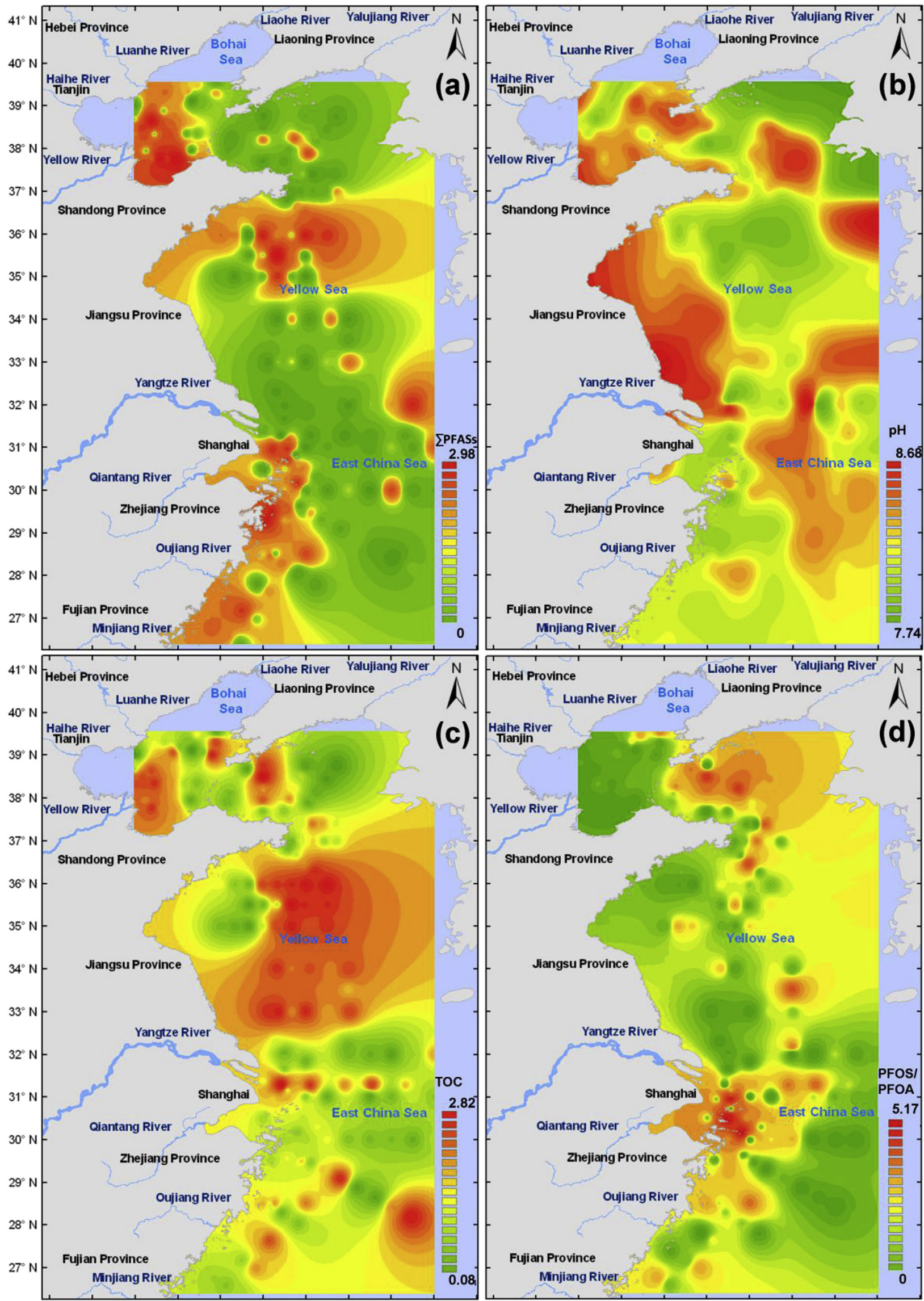

$117^{\circ} \mathrm{E} 118^{\circ} \mathrm{E} 119^{\circ} \mathrm{E} 120^{\circ} \mathrm{E} 121^{\circ} \mathrm{E} 122^{\circ} \mathrm{E} 123^{\circ} \mathrm{E} 124^{\circ} \mathrm{E} 125^{\circ} \mathrm{E} 126^{\circ} \mathrm{E} 127^{\circ}$

$118^{\circ} \mathrm{E} 119^{\circ} \mathrm{E} 120^{\circ} \mathrm{E} 121^{\circ} \mathrm{E} 122^{\circ} \mathrm{E} 123^{\circ} \mathrm{E} 124^{\circ} \mathrm{E} 125^{\circ} \mathrm{E} 126^{\circ} \mathrm{E}$

Fig. 2. Spatial distributions of (a) total PFASs (ng $\mathrm{g}^{-1} \mathrm{dw}$ ), (b) sediment $\mathrm{pH}$, (c) sediment TOC, and (d) PFOS/PFOA ratio. 
hydrophobic chemicals, adsorption to particles tends to be higher in the finer fractions due to their larger surface area to mass ratio (Zhao et al., 2012). Similar observations have been reported for dichlorodiphenyltrichloroethanes (DDTs), hexachlorocyclohexanes (HCHs) and short chain chlorinated paraffins (SCCPs) in the same general study area (Hu et al., 2011; Zeng et al., 2013).

Available data in PFASs concentrations in surface sediments worldwide are compared in Fig. 3. The comparison suggests that the PFASs pollution of the study area is moderate on a global perspective. For PFOA, the concentrations found in this work were higher than those from previous studies from coastal regions of the northern Bohai Sea in China (Chen et al., 2011), comparable to those from the German Bight of the North Sea (Theobald et al., 2012), the west coast of Korea (Naile et al., 2013), and Cantabrian Sea in northern Spain (Gómez et al., 2011), but lower than those reported from Indonesian coastal waters (Harino et al., 2012). The PFOS concentrations in these three sea areas were higher than those in Daliao River of northeast China (Bao et al., 2009; Yang et al., 2011), Taihu Lake (Yang et al., 2011), and rivers in Shanghai (Bao et al., 2010), comparable to those in Baiyangdian Lake of northern China (Shi et al., 2012), and the Baltic Sea (Theobald et al., 2012), but lower than those in Guangzhou, China (Bao et al., 2010), west coast of Korea (Naile et al., 2013), San Francisco Bay (Higgins et al., 2005), and the German Bight of the North Sea (Theobald et al., 2012).

\subsection{PFASs composition analysis}

Composition and correlation analysis among the different PFASs can be applied as a tracer of the possible sources of PFASs by atmospheric depositions or marine current in the marine ecosystem (Ahrens et al., 2009a; Simcik and Dorweiler, 2005). The dominance of PFOS and PFOA in total PFASs has been previously reported in various environmental matrices (Myers et al., 2012; Zhang et al., 2010). In this study, composition analysis indicated that PFOA was the most dominant compound with the highest concentrations and detection frequencies, followed by PFOS and PFUnDA (Fig. S2). Spearman's correlation analysis indicated that, on a 95\% confidence level, PFOA was positively correlated with PFOS, PFHpA, PFNA, PFDA, and PFUnDA. PFOS was positively correlated with PFHpA, PFNA, PFDA, and PFUnDA (Table S6). The significant positive correlations among these compounds suggest that their contamination levels might be all mainly influenced by human activities.

The PFOS/PFOA ratio was examined in some studies with the intention of identifying source regions (Simcik and Dorweiler, 2005; So et al., 2004). In this work, the PFOS/PFOA ratios in the surface sediments $(N=166)$ are mostly $<1$ across the study area (Table S7), but with the $>1$ values at several spots (Fig. 2d). In the $\mathrm{BS}$, the ratios are all $<1$, and the highest was 0.93 at site $\mathrm{B} 17$, which is closest to the mouth of Liaohe River. PFOA was found more abundant than PFOS in the sediments from Dagu and Chentaizi drainage river in Tianjin (Pan et al., 2011), in agreement with the observation of this work for the sites nearest to Tianjin. However, the opposite was found in Daliao River (Bao et al., 2009; Yang et al., 2011) and the coastal regions of the north and east parts of BS (Chen et al., 2011). In the YS, average PFOS/PFOA ratio is statistically higher than that in BS $(p<0.05)$ but still below 1 . Higher ratios were found in the northern and eastern YS, near Liaoning Province and the west coast of Korean Peninsula, where PFOS was previously reported dominant over PFOA (Naile et al., 2013). In the northern region, the ratio tends to increase from south to north and in the southern region the increasing trend was from west to east. In the ECS, the ratio PFOS/PFOA is generally higher than in the other two seas, with a decreasing trend from the coastal mud areas to the opensea. The concentration of PFOS was higher than PFOA in river sediments from Shanghai (Bao et al., 2010), which is consistent with the finding of this work for nearshore sampling sites such as E54, E59, etc. These results demonstrate a general spatial distribution pattern featuring higher PFOS/PFOA ratio closer to sources. Comparing the two chemicals, PFOA is more prone to migration with ocean current whereas PFOS has higher affinity to particles and sediments (Higgins and Luthy, 2006).

Young et al. indicated that PFDA and PFUnDA found in the environment came from oxidation of PFASs precursors, because their productions are very limited (Young et al., 2007). The high detection frequencies of PFDA and PFUnDA in the sediments of this study (Table 1) implied that the indirect sources such as the atmospheric oxidation of PFASs precursors or other processes might be sources of the PFASs in the sediments of the study area.

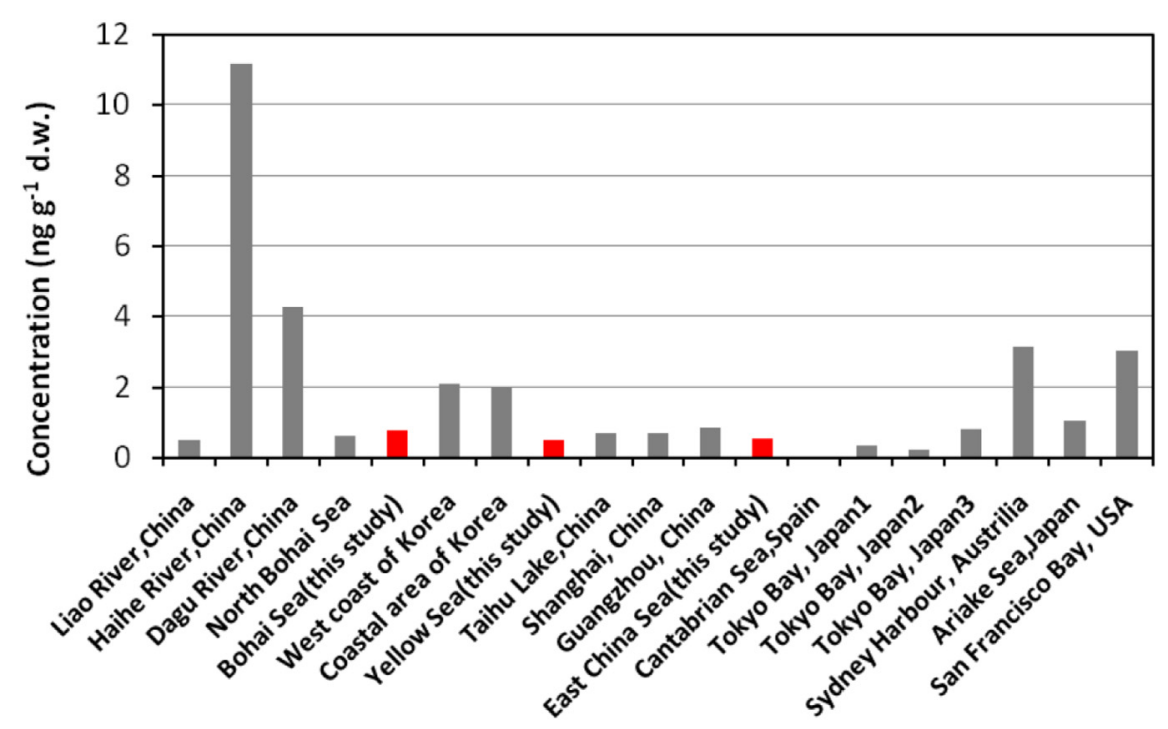

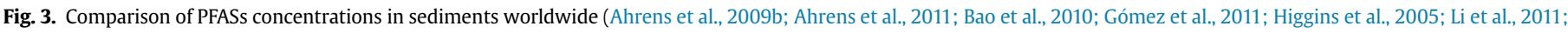
Naile et al., 2013; Naile et al., 2010; Nakata et al., 2006; Sakurai et al., 2010; Thompson et al., 2011; Wang et al., 2011; Yang et al., 2011). 


\subsection{Influencing factors for PFASs distribution}

The TOC and pH of the sediment samples, and the longitude and latitude of the sampling locations were examined individually and in combination for their correlation with the PFASs concentrations. Table S8 showed the results of single-variable regressions. The sediment $\mathrm{pH}$ ranged from 7.70 to 8.70 . The dependence of concentrations on sediment $\mathrm{pH}$ is highly significant for all individual PFASs and $\sum{ }_{9}$ PFASs (Table S8). It was suggested that the $\mathrm{pH}$ can change the organic matter surface charge thus influence the PFASs distributions and the accumulations in the sediments (Ahrens et al., 2009b; Higgins and Luthy, 2006). For example, Higgins et al. have found increasing sorption of PFASs with decreasing $\mathrm{pH}$ of approximately $0.37 \mathrm{log}$ units per unit $\mathrm{pH}$ in the $\mathrm{pH}$ range of 5.7-7.5 (Higgins and Luthy, 2006), and Ahrens et al. pointed out that concentrations of PFOS, PFNA, and PFDoDA in sediment cores increased with the decreasing pH value (Ahrens et al., 2009b). Sediment TOC ranged from 0.08 to 2.82 . TOC normalized concentrations in surface sediments had similar spatial distributions as those based dry weight (Fig. S3). Linear and positive regressions of PFASs on TOC were moderate in general, and appeared to be compound and location dependent (Table S8). The weaker dependence of PFASs on TOC compared with pH suggests that the hydrophobicity is less influential than ionization in affecting PFASs sorption. Dependence on the latitude and longitude of the locations has also been identified between Germany and South Africa in the marine atmosphere (Jahnke et al., 2007), and in the seawater from Atlantic Ocean and the Canadian Arctic Ocean (Benskin et al., 2012). In this work, the dry weight based PFOA concentration was found to decrease with the latitude $(p<0.01)$ and longitude $(p=0.04)$ in the
BS, while in the entire coverage of study area, PFOA decreased with longitude but increased with latitude.

Multivariate regression analyses were carried out to examine the combined impact of the four independent variables including longitude, latitude, $\mathrm{pH}$ and TOC. Table S9 summarizes those that are statistically significant $(p<0.05)$. Take $\sum_{9}$ PFASs in the whole region as an example. The model suggests that $\mathrm{pH}$ and longitude were the key factor that influenced the spatial distribution of PFASs in the marine environmental in China, which accounted for $29 \%$ of the variation in the concentrations of $\sum$ PFASs in the sediments (Table S9). The predicted concentrations using the multi-variable models were compared with the measured concentrations (Fig. 4). On the whole, the most important factor influencing the spatial distributions of PFASs in the sediments is $\mathrm{pH}$, followed by the longitude. In the BS, $\sum_{9}$ PFASs were influenced by TOC. PFOA was influenced by latitude and $\mathrm{pH}$, whereas PFOS was mainly controlled by $\mathrm{pH}$. In YS, ${ }_{9}$ PFASs, PFOS and PFOA were mainly controlled by $\mathrm{pH}$ of the sediments. In ECS, $\sum{ }_{9}$ PFASs and PFOA were jointly controlled by $\mathrm{pH}$ and TOC and PFOS was mainly affected by pH (Fig. 4, Table S9).

The characteristics of PFASs such as chain length and their functional groups can also be the important factors for their distribution. For example, $\mathrm{CF}_{2}$ moiety could increase the sorption to sediment materials for both the PFCAs and the PFSAs. (Higgins and Luthy, 2006).

\subsection{Temporal trends of PFASs from sediment cores}

Sediment cores can preserve the historical records and provide the valuable temporal trends of pollutants in the marine
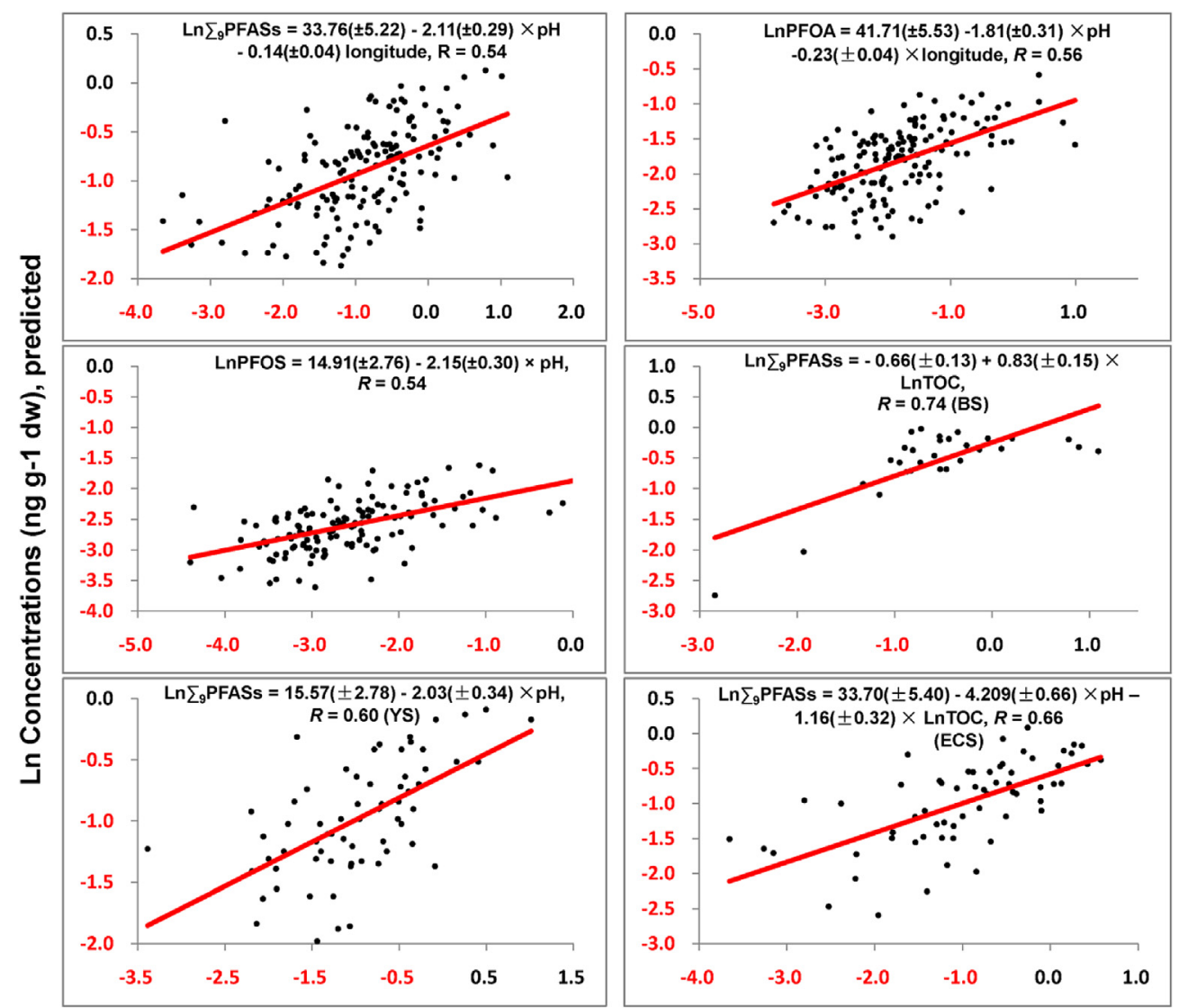

Ln Concentrations (ng $\mathrm{g}^{-1} \mathrm{dw}$ ), measured

Fig. 4. Comparison of the predicted concentrations using the multi-variable models with the measured concentrations. 
environment (Zushi et al., 2010). In this study, 3 sediment cores were collected from the mud areas (Fig. 1, Fig. S1). The concentrations of $\sum{ }_{9}$ PFASs in cores $1,2,3$ were in the range of $<$ LOD-1.21, $<$ LOD-1.65 and $<$ LOD-1.59 $\mathrm{ng} \mathrm{g}^{-1} \mathrm{dw}$, respectively. These were higher than those from Tokyo Bay, Japan (Ahrens et al., 2009b). Generally, PFASs in the 3 sediment cores had similar increasing trends with relatively higher levels in the upper slices than that in the bottom samples (Fig. 5), consistent with the enhanced usage and production in China in recent years. In core-1, the highest concentration of PFASs was found in the top layer $(1-2 \mathrm{~cm})$. A decreasing trend with the sampling depth was found from top layer to the middle sediment segments $(0-17 \mathrm{~cm})$. However, at the deeper sediment segments $(17-37 \mathrm{~cm})$, except the slice with the sampling depth of $17 \mathrm{~cm}$, the core showed a slightly increasing trend. The concentrations of PFASs in the upper layers in core-2 and core- 3 had the higher concentrations than that in core- 1 and the temporal trends of PFASs incores- 2 and 3 had slightly different trends from core- 1 . In core- 2 , concentration of PFASs decreased generally from top to the bottom, and the highest concentration was found in the $6-7 \mathrm{~cm}$. In core-3, concentrations were also found to decrease from top to the bottom layers, and the highest concentration was found in the $9-10 \mathrm{~cm}$. The increasing concentrations of PFASs in the sediment cores hinted the inputs of PFASs to the sampling areas are increasing.

Composition analysis indicated PFOS and PFOA were the predominant pollutants among the targeted compounds in the core samples. Different from the concentrations in surface sediments, PFBS were detected in more than $60 \%$ of the core samples. In core-1, PFOS, PFOA, PFNA and PFUnDA concentrations were significantly negatively related to depth, with increasing trends from the bottom to top layer; whereas no such trends were evident for PFBS, PFHxS, PFHpA, PFDA and PFDoDA. In core-2, PFHxS, PFOA, PFNA, PFDA and PFUnDA showed significantly negatively relationship with depth $(p<0.05)$ whereas in core-3, only PFOA and PFNA concentrations were significantly negatively related to depth.

The temporal trends of PFASs in sediment cores have been reported in the worldwide. Bao et al. found overall decreasing trend along depth in sediment cores from Daliao River system, Zhujiang River andthe Huangpu River (Bao et al., 2009, 2010). Zushi et al. found that a gradual decrease for PFOS after the early 1990s in the sediment core of Tokyo Bay, Japan (Zushi et al., 2010), which was different from the results in our work. Ahrens et al. revealed that $\sum$ PFASs concentrations in the sediment core samples from Tokyo Bay generally increased from bottom to the top but got lower close to the surface (Ahrens et al., 2009b). Myers et al. indicated that the concentrations of PFOS in Niagara River suspended solids decreased during the period of 2001-2006 while that of PFOA showed an increasing trend, but PFOS deposition increased in Lake Ontario cores (Myers et al., 2012). Yeung et al. found that temporal fluxes of PFOS generally increased from 1950s to 2005 in Lake Ontario (Yeung et al., 2013). Related to different production and usage histories in different regions of the world might be responsible for the temporal variation.

Environmental releases of $\mathrm{C} 8$-chained PFASs are expected to decrease dramatically and concomitantly (Paul et al., 2008). Human exposure to these compounds has decreased, which has resulted in the decrease in the human blood or serum with half-lives $4.2-8.2$ years (Glynn et al., 2012; Olsen et al., 2012). While in the environment especially in the remote area such as the Arctic, inflection point for the temporal trend of PFASs may be postponed due to the slow reductions in the current "reservoir" (such as in the ocean water, marine sediment, etc) and continuous long range transport of PFASs and their precursors to the remote areas and oceans (Nash et al., 2010). A survey on the source identification and emission estimation of PFOS in China indicated that the production in China increased rapidly from 2001 to 2006 and kept constant from 2007 to 2011 for some acceptable purpose even after PFOS and its related salt were listed into Stockholm Convention in 2009 (Xie et al., 2013). PFOS emission density in eastern areas is significantly higher than that in the western areas, especially along the coasts (Xie et al., 2013). Some evaluations on the PBT (Persistence, bioaccumulation, and toxicity) of PFOS alternative have been sponsored by the government to reduce the production of PFOS and its related product. However, a detailed inventory for the product, usage of PFOS and PFOS-based product in the Chinese market is still needed to better understand the overall emissions inventory scenario to the environment.

\section{Conclusion}

This study traced the spatial and temporal distribution patterns of PFASs in sediments from BS, YS and ECS. PFASs concentrations in the study region were moderate on a global perspective. Spatial distribution showed a general decreasing trend of PFASs from the coast areas to the open sea. Ocean current might be the dominant source of PFASs to the sediment and the most important factor influencing the spatial distributions of PFASs in the sediments was $\mathrm{pH}$, followed by the longitude. The results help us gain insights into the long range transport of PFASs. The increasing trend of PFASs concentrations from the lower to the upper layers corresponds well

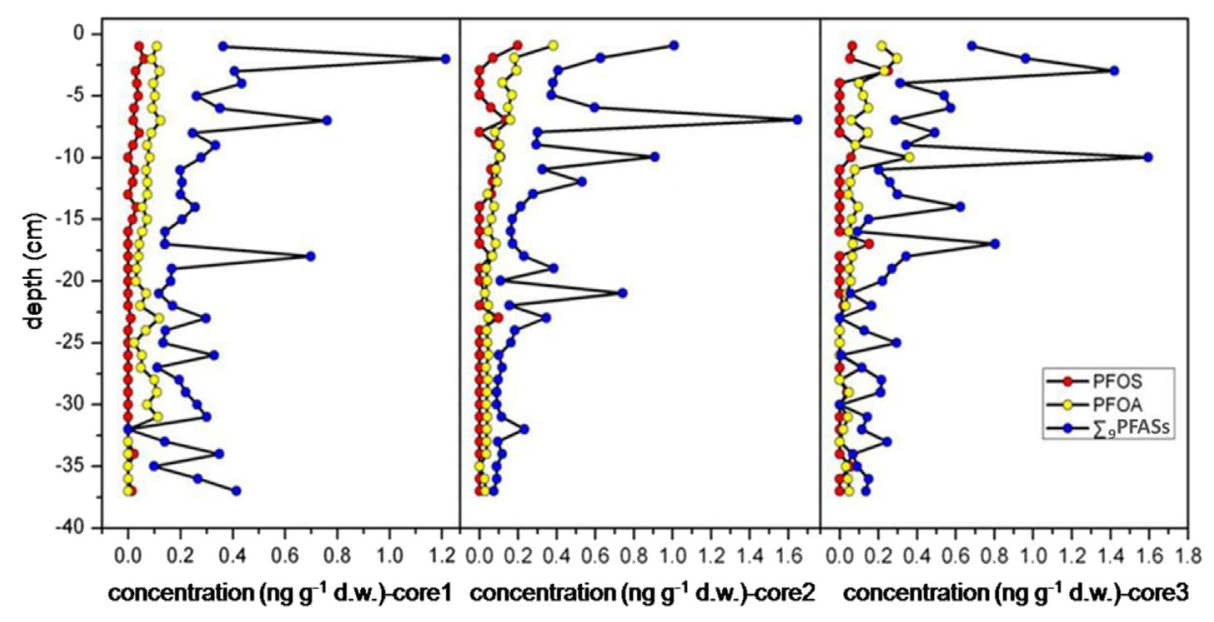

Fig. 5. Temporal trends of PFOS, PFOA and $\sum_{9}$ PFASs in the three sediment cores. 
to the increasing production and usage in China in recent years, necessitating future studies on the impacts on the ecosystem and on human health.

\section{Acknowledgments}

This work was jointly supported by the projects of National Natural Science Foundation (21222702, 21361140359) and Special Fund for Public Service Sector of National Environmental Protection Ministry of China (201009026).

\section{Appendix A. Supplementary data}

Supplementary data related to this article can be found at http:// dx.doi.org/10.1016/j.envpol.2014.07.018.

\section{References}

Ahrens, L. Barber, J.L, Xie, Z, Ebinghaus, R, 2009a. Longitudinal and latitudina distribution of perfluoroalkyl compounds in the surface water of the Atlantic ocean. Environ. Sci. Technol. 43, 3122-3127.

Ahrens, L., Yamashita, N., Yeung, L.W.Y., Taniyasu, S., Horii, Y., Lam, P.K.S. Ebinghaus, R., 2009b. Partitioning behavior of per- and polyfluoroalkyl compounds between pore water and sediment in two sediment cores from Tokyo Bay, Japan. Environ. Sci. Technol. 43, 6969-6975.

Ahrens, L., Yeung, L.W., Taniyasu, S., Lam, P.K., Yamashita, N., 2011. Partitioning of perfluorooctanoate (PFOA), perfluorooctane sulfonate (PFOS) and perfluorooctane sulfonamide (PFOSA) between water and sediment. Chemosphere $85,731-737$

Bao, J., Jin, Y., Liu, W., Ran, X., Zhang, Z., 2009. Perfluorinated compounds in sediments from the Daliao River system of northeast China. Chemosphere 77, $652-657$.

Bao, J., Liu, W., Liu, L., Jin, Y., Ran, X., Zhang, Z, 2010. Perfluorinated compounds in urban river sediments from Guangzhou and Shanghai of China. Chemosphere $80,123-130$

Benskin, J.P., Muir, D.C.G., Scott, B.F., Spencer, C., De Silva, A.O., Kylin, H., Martin, J.W. Morris, A, Lohmann, R. Tomy, G, Rosenberg B, Taniyasu, S, Yamashita, N. 2012. Perfluoroalkyl acids in the Atlantic and Canadian arctic oceans. Environ. Sci. Technol. 46, 5815-5823.

Cai, M., Zhao, Z., Yang, H., Yin, Z., Hong, O., Sturm, R., Ebinghaus, R., Ahrens, L, Cai, M., He, J., Xie, Z., 2012a. Spatial distribution of per- and polyfluoroalkyl compounds in coastal waters from the East to South China Sea. Environ. Pollut. $161,162-169$.

Cai, M., Zhao, Z., Yin, Z., Ahrens, L., Huang, P., Cai, M., Yang, H., He, J., Sturm, R., Ebinghaus, R., Xie, Z., 2012b. Occurrence of perfluoroalkyl compounds in surface waters from the North Pacific to the Arctic Ocean. Environ. Sci. Technol. 46 $661-668$.

Chen, C., Lu, Y., Zhang, X., Geng, J., Wang, T., Shi, Y., Hu, W., Li, J., 2009. A review of spatial and temporal assessment of PFOS and PFOA contamination in China. Chem. Ecol. 25, 163-177.

Chen, C., Wang, T., Khim, J.S., Luo, W., Jiao, W., Lu, Y., Naile, J.E., Hu, W., Zhang, X Geng, J., Bi, C., Li, J., Giesy, J.P., 2011. Perfluorinated compounds in water and sediment from coastal regions of the northern Bohai Sea, China. Chem. Ecol. 27 $165-176$.

Cheng, P., Gao, S., Bokuniewicz, H., 2004. Net sediment transport patterns over the Bohai Strait based on grain size trend analysis. Estuar. Coast. Shelf Sci. 60, $203-212$.

Ellis, D.A., Martin, J.W., De Silva, A.O., Mabury, S.A., Hurley, M.D., Andersen, M.P.S. Wallington, T.J., 2004. Degradation of fluorotelomer alcohols: a likely atmospheric source of perfluorinated carboxylic acids. Environ. Sci. Technol. 38, 3316-3321.

Ellis, D.A., Martin, J.W., Mabury, S.A., Hurley, M.D., Andersen, M.P.S., Wallington, T.J. 2003. Atmospheric lifetime of fluorotelomer alcohols. Environ. Sci. Technol. 37, 3816-3820.

Gómez, C., Vicente, J., Echavarri-Erasun, B., Porte, C., Lacorte, S., 2011. Occurrence of perfluorinated compounds in water, sediment and mussels from the Cantabrian Sea (North Spain). Mar. Pollut. Bull. 62, 948-955.

Glynn, A., Berger, U., Bignert, A., Ullah, S., Aune, M., Lignell, S., Darnerud, P.O., 2012. Perfluorinated alkyl acids in blood serum from primiparous women in Sweden: serial sampling during pregnancy and nursing, and temporal trends 1996-2010. Environ. Sci. Technol. 46, 9071-9079.

Harino, H., Arifin, Z., Rumengan, I.F.M., Arai, T., Ohji, M., Miyazaki, N., 2012. Distribution of antifouling biocides and perfluoroalkyl compounds in sediments from selected locations in indonesian coastal waters. Arch. Environ. Contam. Toxicol. $63,13-21$.

Higgins, C.P., Field, J.A., Criddle, C.S., Luthy, R.G., 2005. Quantitative determination of perfluorochemicals in sediments and domestic sludge. Environ. Sci. Technol. 39, 3946-3956.
Higgins, C.P., Luthy, R.G., 2006. Sorption of perfluorinated surfactants on sediments. Environ. Sci. Technol, 40, 7251-7256.

Hu, L.M., Lin, T., Shi, X.F., Yang, Z.S., Wang, H.J., Zhang, G., Guo, Z.G., 2011. The role of shelf mud depositional process and large river inputs on the fate of organochlorine pesticides in sediments of the Yellow and East China seas. Geophys. Res. Lett. 38.

Jahnke, A., Berger, U., Ebinghaus, R., Temme, C., 2007. Latitudinal gradient of airborne polyfluorinated alkyl substances in the marine atmosphere between Germany and South Africa (53 degrees N-33 degrees S). Environ. Sci. Technol. 41, 3055-3061.

Kelly, B.C., Ikonomou, M.G., Blair, J.D., Surridge, B., Hoover, D., Grace, R., Gobas, F.A.P.C., 2009. Perfluoroalkyl contaminants in an arctic marine food web: trophic magnification and wildlife exposure. Environ. Sci. Technol. 43, 4037-4043.

Li, F., Sun, H., Hao, Z., He, N., Zhao, L., Zhang, T., Sun, T., 2011. Perfluorinated compounds in Haihe river and dagu drainage canal in tianjin, China. Chemosphere 84, 265-271.

Li, J., Guo, F., Wang, Y., Zhang, J., Zhong, Y., Zhao, Y., Wu, Y., 2013. Can nail, hair and urine be used for biomonitoring of human exposure to perfluorooctane sulfonate and perfluorooctanoic acid? Environ. Int. 53, 47-52.

Li, J., Huang, X., Li, Y., 2005. Determine pH value of the marine sediment with different methods. Instrum. Anal. Monit. (in Chinese) 3, 33.

Liu, J., Li, A., Xu, K., Velozzi, D., Yang, Z., Milliman, J., DeMaster, D., 2006. Sedimentary features of the Yangtze River-derived along-shelf clinoform deposit in the East China Sea. Cont. Shelf Res. 26, 2141-2156.

Liu, J., Xu, K., Li, A., Milliman, J.D., Velozzi, D.M., Xiao, S.B., Yang, Z.S., 2007. Flux and fate of Yangtze river sediment delivered to the East China Sea. Geomorphology 85, 208-224.

Loi, E.I.H., Yeung, L.W.Y., Taniyasu, S., Lam, P.K.S., Kannan, K., Yamashita, N., 2011. Trophic magnification of poly- and perfluorinated compounds in a subtropical food web. Environ. Sci. Technol. 45, 5506-5513.

Myers, A.L., Crozier, P.W., Helm, P.A., Brimacombe, C., Furdui, V.I., Reiner, E.J., Burniston, D., Marvin, C.H., 2012. Fate, distribution, and contrasting temporal trends of perfluoroalkyl substances (PFASs) in Lake Ontario, Canada. Environ. Int. 44, 92-99.

Naile, J.E., Khim, J.S., Hong, S., Park, J., Kwon, B.-O., Ryu, J.S., Hwang, J.H., Jones, P.D., Giesy, J.P., 2013. Distributions and bioconcentration characteristics of perfluorinated compounds in environmental samples collected from the west coast of Korea. Chemosphere 90, 387-394.

Naile, J.E., Khim, J.S., Wang, T., Chen, C., Luo, W., Kwon, B.-O., Park, J., Koh, C.-H., Jones, P.D., Lu, Y., Giesy, J.P., 2010. Perfluorinated compounds in water, sediment, soil and biota from estuarine and coastal areas of Korea. Environ. Pollut. 158, 1237-1244.

Nakata, H., Kannan, K., Nasu, T., Cho, H.-S., Sinclair, E., Takemura, A., 2006. Perfluorinated contaminants in sediments and aquatic organisms collected from shallow water and tidal flat areas of the Ariake Sea, Japan: environmental fate of perfluorooctane sulfonate in aquatic ecosystems. Environ. Sci. Technol. 40, 4916-4921.

Nash, S.B., Rintoul, S.R., Kawaguchi, S., Staniland, I., van den Hoff, J., Tierney, M., Bossi, R., 2010. Perfluorinated compounds in the Antarctic region: ocean circulation provides prolonged protection from distant sources. Environ. Pollut. 158, 2985-2991.

Olsen, G.W., Lange, C.C., Ellefson, M.E., Mair, D.C., Church, T.R., Goldberg, C.L. Herron, R.M., Medhdizadehkashi, Z., Nobiletti, J.B., Rios, J.A., 2012. Temporal trends of perfluoroalkyl concentrations in American Red Cross adult blood donors, 2000-2010. Environ. Sci. Technol. 46, 6330-6338.

Pan, Y., Shi, Y., Wang, J., Jin, X., Cai, Y., 2011. Pilot investigation of perfluorinated compounds in river water, sediment, soil and fish in Tianjin, China. Bull. Environ. Contam. Toxicol. 87, 152-157.

Pan, Y., Shi, Y., Wang, Y., Cai, Y., Jiang, G., 2010. Investigation of perfluorinated compounds (PFCs) in mollusks from coastal waters in the Bohai Sea of China. J. Environ. Monit. 12, 508-513.

Paul, A.G., Jones, K.C., Sweetman, A.J., 2008. A first global production, emission, and environmental inventory for perfluorooctane sulfonate. Environ. Sci. Technol. 43, 386-392.

Prevedouros, K., Cousins, I.T., Buck, R.C., Korzeniowski, S.H., 2006. Sources, fate and transport of perfluorocarboxylates. Environ. Sci. Technol. 40, 32-44.

Sakurai, T., Serizawa, S., Isobe, T., Kobayashi, J., Kodama, K., Kume, G., Lee, J.-H., Maki, H., Imaizumi, Y., Suzuki, N., 2010. Spatial, phase, and temporal distributions of perfluorooctane sulfonate (PFOS) and perfluorooctanoate (PFOA) in Tokyo Bay, Japan. Environ. Sci. Technol. 44, 4110-4115.

Shi, Y., Pan, Y., Wang, J., Cai, Y., 2012. Distribution of perfluorinated compounds in water, sediment, biota and floating plants in Baiyangdian Lake, China. J. Environ. Monit. 14, 636-642.

Simcik, M.F., Dorweiler, K.J., 2005. Ratio of perfluorochemical concentrations as a tracer of atmospheric deposition to surface waters. Environ. Sci. Technol. 39, 8678-8683.

So, M.K., Taniyasu, S., Yamashita, N., Giesy, J.P., Zheng, J., Fang, Z., Im, S.H., Lam, P.K.S., 2004. Perfluorinated compounds in coastal waters of Hong Kong, South China, and Korea. Environ. Sci. Technol. 38, 4056-4063.

Theobald, N., Caliebe, C., Gerwinski, W., Huhnerfuss, H., Lepom, P., 2012. Occurrence of perfluorinated organic acids in the North and Baltic Seas. Part 2: distribution in sediments. Environ. Sci. Pollut. Res. 19, 313-324.

Thompson, J., Roach, A., Eaglesham, G., Bartkow, M.E., Edge, K., Mueller, J.F., 2011. Perfluorinated alkyl acids in water, sediment and wildlife from Sydney harbour and surroundings. Mar. Pollut. Bull. 62, 2869-2875. 
Wang, T., Lu, Y., Chen, C., Naile, J.E., Khim, J.S., Park, J., Luo, W., Jiao, W., Hu, W., Giesy, J.P., 2011. Perfluorinated compounds in estuarine and coastal areas of north Bohai Sea, China. Mar. Pollut. Bull. 62, 1905-1914.

Xie, S., Wang, T., Liu, S., Jones, K.C., Sweetman, A.J., Lu, Y., 2013. Industrial source identification and emission estimation of perfluorooctane sulfonate in China. Environ. Int. 52, 1-8.

Yanao, S., Matsuno, T., 2013. Characteristics of outer shelf water in the East China Sea. J. Oceanogr. 69, 245-258.

Yang, L. Zhu, L., Liu, Z., 2011. Occurrence and partition of perfluorinated compounds in water and sediment from Liao River and Taihu Lake, China. Chemosphere 83 $806-814$.

Yeung, L.W., De Silva, A.O., Loi, E.I., Marvin, C.H., Taniyasu, S., Yamashita, N., Mabury, S.A., Muir, D.C., Lam, P.K., 2013. Perfluoroalkyl substances and extractable organic fluorine in surface sediments and cores from Lake Ontario. Environ. Int. 59, 389-397.
Young, C.J., Furdui, V.I., Franklin, J., Koerner, R.M., Muir, D.C.G., Mabury, S.A., 2007. Perfluorinated acids in arctic snow: new evidence for atmospheric formation. Environ. Sci. Technol. 41, 3455-3461.

Zeng, L., Chen, R., Zhao, Z., Wang, T., Gao, Y., Li, A., Wang, Y., Jiang, G., Sun, L., 2013. Spatial distributions and deposition chronology of short chain chlorinated paraffins in marine sediments across the Chinese Bohai and Yellow Seas. Environ. Sci. Technol. 47, 11449-11456.

Zhang, T., Sun, H.W., Wu, Q., Zhang, X.Z., Yun, S.H., Kannan, K., 2010. Perfluorochemicals in meat, eggs and indoor dust in China: assessment of sources and pathways of human exposure to perfluorochemicals. Environ. Sci. Technol. 44, 3572-3579.

Zhao, L., Zhu, L., Yang, L., Liu, Z., Zhang, Y., 2012. Distribution and desorption of perfluorinated compounds in fractionated sediments. Chemosphere 88, 1390-1397.

Zushi, Y., Tamada, M., Kanai, Y., Masunaga, S., 2010. Time trends of perfluorinated compounds from the sediment core of Tokyo Bay, Japan (1950s-2004). Environ. Pollut. 158, 756-763. 